the beginning of September, the oxidizing influence of the air, as tested by ozone paper, has been distinct, a character which was absent in September, 1854, and is an evidence, if of nothing else, at least of the prevalence of less stagnation of the atmosphere of London in the present than in the previous summer. This result seems worthy of notice, since a deficiency in the atmospheric motion appears to be an accompaniment of cholera, if we may judge from the plenomena observed during the recent occurrence of the epidemic, both in this country and in Germany. In the last report it was stated that no symptoms of the same condition of the air, which prevailed in 1854, had then exhibited themselves; such, at least, as to lead to the inference of the existence of a cholera atmosphere, and since that period nothing has occurred to lead to a change of the opinion then expressed.

\section{REPORT OF A \\ CASE OF SUDDEN DEATH OCCURRING DURING LABOUR.}

By GEORGE ROPER, Esq., M.R.C.S., L.S.A.

ON the 5th of April, 1854, M. A. K-, aged thirty-eight, was taken in labour with her first child. In the early part of labour, her pains were but slight, and she had been engaged in the performance of her domestic duties till within two hours and $a$ half before her death. The feet of the child presented; beyond this, there was nothing remarkable. For the labour of a primipara, at the age of thirty-eight, it was a very easy one. The pelvis was capacious, the soft parts were free from rigidity, and the passage of the child met with no obstruction. She had not been placed in bed more than half an hour before the trunk and arms of the child were delivered. Up to this point of her labour, she did not complain, nor had she exhibited any symptoms of distress; but just as the foetal head was distending the perinæum, and about to pass, she raised herself from the pillow, and exclaimed that she should be choked. It was immediately perceived that she was in a state of asphyxia. The face was swollen, and of a deep livid colour; the lips were purple; she made gasping efforts to breathe; the nostrils became widely expanded; the countenance assumed an expression of impending suffocation; a stream of frothy mucus issued from the mouth and nose; and she was dead in three minutes after these symptoms were first observed. Death occurred too speedily to allow of much treatment. The head and shoulders were raised, so as to put the body in a semi-erect posture; and with a view of relieving the congestion, a vein was freely opened in each arm, but no blood could be obtained. It was utterly impossible that the patient could swallow anything; she was, in fact, moribund at the time her condition was discovered. Although life had ceased, it was thought prudent and desirable that a physician-acconcheur should be summoned as a witness of such an unusual occurrence. A messenger was sent for Dr. Oldham or Dr. Winn. The latter gentleman, who was at home, kindly came, and in his presence, withont the least difficulty, I removed the foetal head, which had remained undelivered. It requires to be explained, that when the alarming symptoms were noticed, it was feared that any forcible effort to extract the fotal head would render the mother's condition, if possible, more perilous.

A post-mortem examination of the body was made eleven hours after death, at which Dr. Winn was present. There were no morbid appearances in the brain, beyond an unnatural turgescence of its bloodvessels. In the chest, the large veins and the superior cava were found greatly distended, as was, also, the right auricle. The lungs were enormously congested; they were gorged with blood, and, from their density and heaviness, more resembled a sponge saturated with flnid than the natural lightness of pulmonary texture. A small, lightcoloured fringe at the base of the left lung, where there was a pleuritic adhesion, was the only part free from congestion. The right auricle, as has been stated, was enormously distended with blood; the right ventricle was equally so. The cavity of each was greatly dilated; their muscular structure was flabby and attenuated. The left ventricle was unusually thick and strong; its cavity was empty, and not enlarged. There was no valvular disease. The coronary arteries were unobstructed. The aorta and pulmonary artery were healthy. The abdominal viscera were all healthy, but exhibited a high degree of congestion. Nothing unusual was observed in the pelvic organs. The blood was everywhere thin, free from clot, and of dark colour.

By post-mortem inspection, two conditions of chief import- ance were observed:-Firstly, the gorged pulmonary circulation, including the cavities of the right heart. Secondly, the attenuated and flabby muscular tissue of the right heart. The altered state of respiration during labour fully explains how this congestion happened. In ordinary inspiration, the blood becomes retarded in its passage through the julmonary circulation. During forced inspiration, greater retardation takes place; the pulmonary artery, and the cavities of the right heart, become unusually distended. Now, this occurs in an eminent degree during a parturient throe, in which not only is a deep inspiration taken, but a violent straining effort is made with closed glottis, a condition of respiration leading to great obstruction of pulmonary circulation, and a corresponding accumulation in the cavities of the right heart. The fatal amount of congestion of the pulmonary circulation which existed in the present case is readily accounted for by the state of respiration during labour, and the thin, dilated right heart, causes which acted and re-acted on each other. Unable to expel and force onwards its contents, and continually filled with blood, poured into it from the systemic circulation, the right heart became choked, and its action arrested. It is regretted that no microscopic examination of the tissue of the heart was made; but whatever might have been the pathological condition of its muscular fibre, the appearances of the organ, to the unaided eye, afforded conclusive evidence of its defective and inadequate power. The patient was a welldeveloped subject, rather muscular, and not fat. Prior to her marriage, two years since, her health had not been good. She had always been ailing, but had never had any very severe illness. Since her marriage she had had much better health. During life, she had on each cheek a purplish, circumscribed patch, consisting of enlargement of the capillaries or very small bloodvessels, showing a bad state of venous circulation. September, 1855 .

$O N$

\section{AN ANOMALOUS CASE OF SCARLATINA.}

\section{BY WM. WHXTEHEAD MORRIS, L.S.A., \&c.}

Tre following case, which has lately occurred in my practice, presenting many deviations from what I have previonsly seen, heard, or read of, in scarlatina, I am induced to lay the particulars before my professional brethren, and to ask if they have recently or ever observed similar cases.

Elizabeth F- aged six years and ten months, romited, and complained of headache on Friday evening, March 2 nd. On the 4th I was requested to visit her. An eruption had been taking place during the day on the face and extremities, particularly the latter, and nowhere else; it consisted of spots, rosy in colour, one-sixth to one-fourth of an inch in diameter, arranged in sigmoid and crescentic patches of one-half of an inch to one inch in length, covering nearly the whole surface of the arms, thighs, and legs, and very evidently raised above the level of the skin; each spot or papula was circular, or nearly so, in form, consisting of a bright ring, about one-twenty-fourth of an inch wide, enclosing a nearly white area, much resembling miniature ringworm. The intervening portions of shin were clear and natural in appearance. Pulse 141-152, smcll, hard, irregular in frequency; much general heat of surface; constant thirst; passive delirium; pupils much dilated, and scarcely sensible to lirht; urize very scanty, pale, and clear; bowels regular; tongue moist, white, and smooth. Take of the nitrate of potash, half a drachm; of the syrup of red popries, three drachms; add water, to three ounces. Dose, three teaspoonfuls every two hours. Take of mercury with chalk, bicarbonate of soda, of each one grain, and make a porrder to be taken with each dose of the mixture; put up six. Rectified spirits of wine, three drachms; coloured water, six ounces: make a lotion, to be applied constantly to the head. Brown's cantharidine, five inches by two, to be applied to the back of the neck for three hours.

March 5th.-Ten A. M. : Has passed a tolerable nicht ; thirst less urgent; pulse 130 , more regular; urine slightly increased in quantity; has roided a frorm (ascaris lumbricoides) with several greenish shreddy stools. Other symptoms as before. Continue the mixture and lotion, and omit the powders.

6th. - Half-past ten A.M.: Was more delirious last evening, but passed a good night; pulse now 120, softer and fuller; intellect much clearer; skin moist; eruption duller, but appearing in no other place than previously indicated; bowels moved three times since last risit; stools natural in appearance; urine still scantier than in health; slight strawberry appearance on the tip of the tongue only, the rest being still white; glands 345 